\title{
PNEUMOMEDIASTINUM AND SUBCUTANEOUS EMPHYSEMA IN STATUS ASTHMATICUS, REQUIRING SURGICAL DECOMPRESSION
}

\author{
BY \\ BRIAN MCNICHOLL \\ From the Regional Hospital, Galway
}

(RECEIVED FOR PUBLICATION NOVEMBER 10, 1959)

Leakage of air into the interstitial tissues of the lungs of sufficient degree to produce pneumomediastinum and subcutaneous emphysema is an uncommon and potentially fatal complication of bronchial asthma in childhood. Another possibly fatal complication of similar causation is tension pneumothorax. It is possible that interstitial emphysema occurs more frequently in severe asthma and allied states than our usual methods of diagnosis allow us to discover, producing additional interference with ventilation by distending and splinting the area of lung involved. This case report and discussion of some relevant literature may serve to illustrate some of these points.

\section{Case Report}

A boy, 8 years of age, had had periodic attacks of asthma for four years, some precipitated by hay making and others by dusting of rooms. He had been admitted to this hospital in November, 1956, on the third day of a severe attack in which there was blood staining of the sputum; the attack responded to intravenous aminophylline and oral ephedrine hydrochloride. Skin tests with Bencards reagents showed marked sensitivity to mixed pollens, house dust, horse hair and cat fur. Between January and March, 1957, hyposensitization with a mixed vaccine was attempted, one brief asthmatic attack occurring during the course. A paternal uncle and a grand-uncle were asthmatic.

The present attack had commenced some 20 hours before his admission on April 14, 1957; he had been given approximately 12 subcutaneous injections of adrenalin by his family doctor and a final injection of $0.5 \mathrm{ml}$. in divided doses by the physician in a district hospital; some ephedrine had been given orally. He had to be brought from an island off the west coast by boat and then 60 miles by road to this hospital. On admission there was marked expiratory and inspiratory dyspnoea, with inspiratory lower costal retraction; the respiratory rate was $55 / \mathrm{min}$., the cardiac rate 160 and the temperature $100^{\circ} \mathrm{F}$. There appeared to be acute-on-chronic pulmonary emphysema and the cardiac dullness was obliterated. The blood pressure was $140 / 90 \mathrm{~mm}$. $\mathrm{Hg}$, the weight $49 \mathrm{lb}$. Small amounts of blood-stained sputum were expectorated with difficulty and cough was forcible and distressing. Aminophylline $0.125 \mathrm{~g}$. was given intravenously on two occasions with temporary relief; 500,000 units of intramuscular penicillin and $\frac{1}{2}$ gr. of phenobarbitone were given, and repeated at 12 hour and six hour intervals respectively.

Four hours after admission subcutaneous emphysema was observed above the clavicles, quickly spreading to the neck, cheeks and axillae; the blood pressure was then $150 / 90 \mathrm{~mm}$. Hg. Three hours later the respiratory distress became more marked and was accompanied by cyanosis despite an efficient oxygen tent. The chest was almost fixed in the maximal inspiratory position, the neck and upper thoracic veins were engorged, the pulse volume was diminished and showed paradoxical respiratory variation.

Cortisone acetate $100 \mathrm{mg}$. was given intramuscularly. Pneumomediastinum was suspected and confirmed by portable radiographs (Figs. 1 and 2). As the boy's condition was critical, horizontal incisions of about $1 \frac{1}{2}$ in. were made under local anaesthesia above each clavicle, about eight and a half hours after admission. Exploration with sinus forceps through the incisions allowed release of air under pressure with marked relief of the distress and of the venous distension. The pulse volume improved and the boy rested comfortably. Gradual resolution of the asthmatic attack followed, the subcutaneous emphysema taking some days to disappear; several exacerbations of the asthma occurred before his discharge nine weeks later. The boy has since been admitted with two severe attacks without recurrence of any observable pulmonary air leak.

\section{Discussion}

Peterson (1947) states that mediastinal emphysema was first described by Müller in 1888, while Matts (1957) states that Laennec (1827) described it under the name of 'interlobular emphysema'.

Macklin and Macklin (1944) discuss the subject of non-surgical interstitial emphysema of the lungs and mediastinum exhaustively, and they maintain 


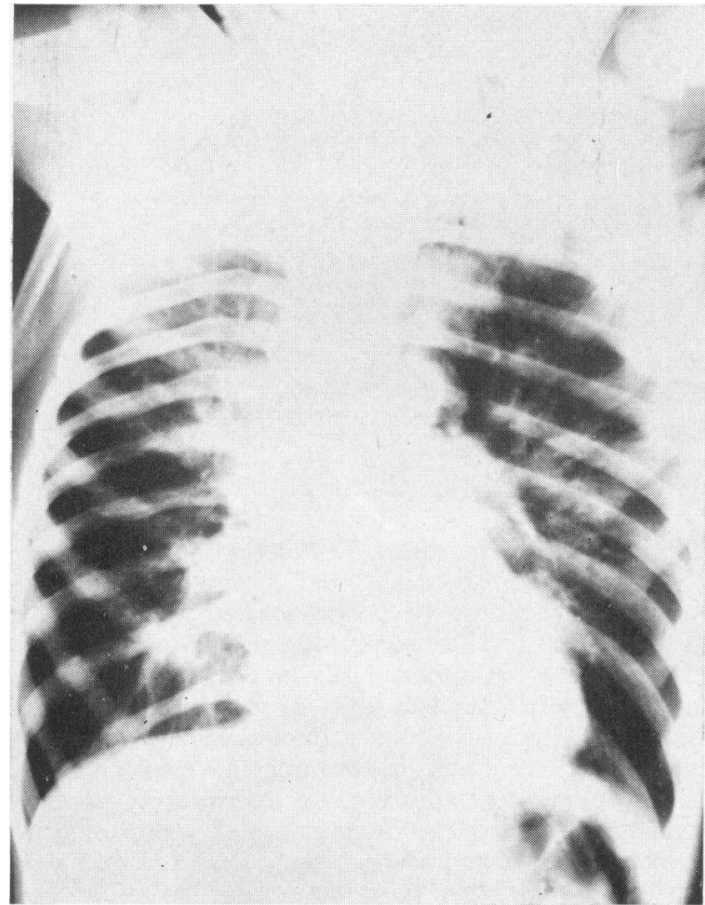

FIG. 1.-Portable antero-posterior film of chest showing distension of lungs, some infiltration of both upper lobes, mainly right, a translucent ring of air surrounding the aortic knuckle and air bubbles in left axilla.

that the basic requirement for alveolar rupture and leakage of air into the interstitial tissues of the lung is a pressure gradient from the alveoli to the perivascular sheaths. The pressure gradient may arise from over-inflation of the lung or lowering of vascular pressure; an example of the former, would be compensatory over-inflation of the lung surrounding an area of atelectasis, and of the latter, would be pulmonary embolism. In the majority of instances of interstitial emphysema there is also an increase in the alveolar pressure. Over-inflation of lung may be localized, as in the lung adjoining an atelectatic area or in lung distal to a ball valve bronchial obstruction. Generalized over-inflation appears to cause most cases of air leak, the most common examples being severe expiratory dyspnoea as in bronchial asthma, forceful coughing, severe straining as in lifting of heavy weights or during labour, other examples being forceful resuscitation of the newborn infant or anaesthetized patient. Other examples of interstitial emphysema in which various factors operate and may combine are neonatal atelectasis, obstructive laryngitis, tracheotomy, pertussis, bronchial foreign bodies,

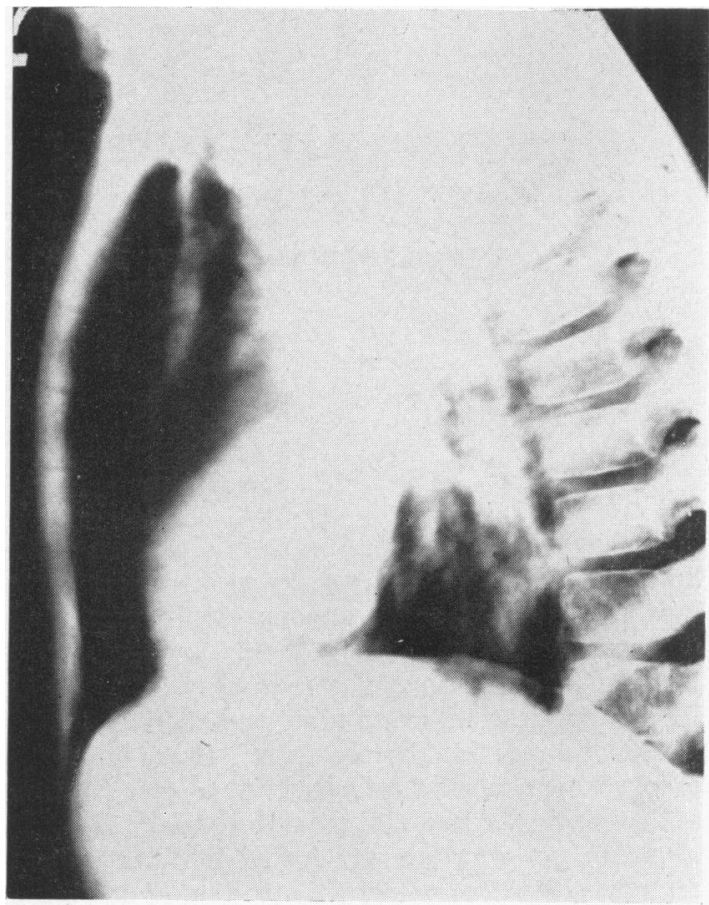

FIG. 2.-Portable lateral film of chest showing large volume of air trapped between heart and sternum, outlining what is probably the thymus. The contrast between the small (presumably compressed) heart and the distended thorax is striking.

severe exercise, lung blast and caisson disease. Pulmonary infection appears to be a factor in many cases, presumably by weakening the alveolar wall, and combined with forceful cough is probably the cause of interstitial emphysema in influenza and measles.

Once air has leaked from the alveolus, if the pressure gradient is maintained, as by persistent dyspnoea and/or coughing, the air usually tracks centrally along the vascular sheaths to the lung root and mediastinum, as Macklin and Macklin (1944) have shown both by experiment and in autopsy material; it may also track peripherally to the subpleural space. The interstitial emphysema may both distend the lung, reducing its mobility and thus interfering with ventilation, and interfere with the pulmonary circulation by compressing blood vessels. When air reaches the mediastinum in large quantities, and does not readily find egress, a tension pneumomediastinum is produced, which impedes venous filling of the heart and thus further restricts the pulmonary, as well as the systemic, circulation; marked venous distension of the neck, head and trunk will occur. Electrocardiographic changes 
have been reported (Master, Kalter and Dack, 1941). To these effects will, in many cases, be added interference with ventilation by local or generalized alveolar emphysema and later by pulmonary infection.

From the mediastinum air may escape into one or both pleural cavities, into the tissues of the neck and thence to the head and trunk, or into the retroperitoneal space from which it may rupture into the peritoneum; escape of air by one or other of these routes usually acts as a safety valve, preventing cardiac tamponade, although the other hazard of tension pneumothorax may be produced. Macklin and Macklin (1944) believe that varying degrees of interstitial emphysema may frequently occur in such conditions as severe asthma and may play a significant part in the dyspnoea; although agreeing with other authors that such leakage of air is usually benign, due either to its limited extent or to the operation of the 'safety valve', they emphasize its potentially fatal nature.

Norman (1957) in describing a boy of 21 months who had a pneumothorax and subcutaneous emphysema complicating bronchial asthma on two occasions, found 30 published cases of subcutaneous emphysema in asthma. Two were fatal, and in two others incision of the skin in the neck to allow escape of air was necessary. Macklin and Macklin (1944) cite reports of other fatal cases of asthma where death was attributable to pneumomediastinum, pneumothorax, or both, in the absence of subcutaneous emphysema.

Recognition and Management. Macklin and Macklin (1944) maintain that the presence of subcutaneous emphysema in the neck presupposes a pneumomediastinum, which should also be suspected in severe asthma without subcutaneous emphysema if there is marked venous distension of the neck, head and upper thorax, or other evidence of cardiac tamponade such as paradoxical pulse with falling blood pressure. In patients old enough to complain, there may be substernal pain of anginal nature, abdominal pain, or a feeling of fullness in the upper chest and neck. Obliteration of cardiac dullness, bulging and tympany of the upper sternum are signs of pneumomediastinum but will be more commonly due to acute generalized pulmonary alveolar emphysema. Systolic crunching or crackling sounds, best heard with the patient in the left lateral position (Hamman, 1937) are said to be diagnostic of air in the mediastinum. Lateral radiograph of the chest is the most valuable procedure, the presence of air between the cardiac and sternal shadows being diagnostic; air may also be seen surrounding the heart in postero-anterior views. Increasing cyanosis and signs of reduced cardiac output, with weakening peripheral circulation will call for urgent decompression of the trapped air in the mediastinum. If air is confined to the mediastinum, needling and under water drainage are indicated. When subcutaneous emphysema is present, incision of the skin in the suprasternal notch is usually adequate, exploration with sinus forceps or gloved finger being continued until sufficient air has been released. Schrire and Schrire (1952) described a 6 in. horizontal incision in the suprasternal notch, vertical incision of the platysma, elevation of the thyroid and insertion of a finger down either side of the trachea to facilitate escape of air, but this procedure should hardly be necessary in most cases. Bilateral incisions, as in the present case, would not seem to be necessary. Following decompression, the wound should be left open and covered with loose gauze, broad spectrum antibiotic cover being provided.

\section{Summary}

Pneumomediastinum and subcutaneous emphysema complicating severe asthma in an 8-year-old boy are described; release of air by means of incisions of the skin in the neck was necessary due to signs of cardiac tamponade.

Some of the relevant literature is reviewed, including an acceptable theory of production of emphysema of interstitial pulmonary tissues, mediastinum and subcutis.

Recognition and management of pneumomediastinum are briefly discussed.

I wish to thank Mr. D. Kneafsey for reading and criticizing this paper and for his help with later interpretation of the radiographs; also Dr. John Horan for his case notes and help with management of the patient.

\section{REFERENCES}

Hamman, L. (1937). Spontaneous interstitial emphysema of the lungs. Trans. Ass. Amer. Phycns, 52, 311

Laennec, R. T. H. (1827). A Treatise on the Diseases of the Chest and on Mediate Auscultation. 2nd ed. Underwood, London.

Macklin, M. T. and Macklin, C. C. (1944). Malignant interstitial emphysema of the lungs and mediastinum as an important occult complication in many respiratory diseases and other conditions: an interpretation of the clinical literature in the light of laboratory experiment. Medicine (Baltimore), 23, 281 .

Master, A. M., Kalter, H. H. and Dack, S. (1941). Spontaneous pneumothorax simulating acute coronary occlusion. $J . M t$ Sinai Hosp., 8, 89.

Matts, S. G. F. (1957). Acute spontaneous mediastinal emphysema. Lancet, 1, 507.

Müller, F. (1888). Ueber Emphysem des Mediastinum. Berl. klin. Wschr., 25, 205.

Norman, M. (1957). Spontaneous pneumothorax and subcutaneous emphysema. Brit. med. J., 2, 1414.

Peterson, H. (1947). A fatal case of bronchial asthma complicated by mediastinal and subcutaneous emphysema. J. Allergy, $18,413$.

Schrire, T. and Schrire, I. (1952). Surgical emphysema in asthma. S. Afr. med. J., 26, 465 . 


\section{Addendum}

A 4-year-old girl was admitted in March, 1960, in the third day of an attack of bronchopneumonia, with subcutaneous emphysema in the neck of six hours' duration. Within a few hours the emphysema had spread to the cheeks, left temple and left lower eyelid, both axillae, and had formed a tense V-shaped swelling over the upper chest with its apex at the sternal angle. There was a forceful dry cough with some expiratory difficulty, wheezing and grunting, suggesting predominant involvement of the bronchioles. Pneumomediastinum was shown by many crunching and bubbling sounds with respiration over the whole sternal area and by very well marked praecordial systolic 'crunching' sounds; the pneumomediastinum was confirmed by radiography. The child was not very distressed at any stage and there was no overfilling of neck veins, but there was thought to be some diminution of pulse volume during inspiration. An interesting finding was regular lowering of the amplitude of the ventricular complexes in the E.C.G. during inspiration, this being most marked in leads $V_{1}$ and $V_{4} R$, without any other abnormal finding. The cardiac rate averaged 150 per minute for the first few hours, and no fall in blood pressure occurred. It is presumed that the E.C.G. findings were due to compression of heart or great vessels, as they had become less marked some days later, and had gone two weeks later.

It was not necessary to decompress the emphysema and this had almost completely disappeared five days later. The respiratory infection responded satisfactorily, intramuscular penicillin being given for eight days. Radiographs showed some pulmonary distension and slight infiltration consistent with a diffuse bronchopneumonia, in addition to air bubbles in soft tissue and mediastinum.

A further interesting feature in connexion with this case is that her brother was admitted to the Fever Department of this hospital in November, 1954, when aged 5 years (under the care of Dr. B. J. O'Driscoll) with subcutaneous and mediastinal emphysema complicating measles bronchopneumonia, the condition subsiding uneventfully with penicillin treatment. The occurrence in siblings of pulmonary air leakage complicating respiratory infections might be due to a common developmental anomaly of the lungs predisposing towards air leakage with certain types of respiratory involvement. It might equally well be coincidental. 